\title{
Psychosis caused by caffeine or a new scientific contribution?
}

Ivana Todorić Laidlaw", Sandra Caratan², Svjetlana Šitum², Vanja Lovretić ${ }^{2}$, Ana Vuksan³, Milena Jukić Bešlić $^{1}$, Berislav Momčilović ${ }^{4}$ Ninoslav Mimica ${ }^{1,5}$

1 University Hospital Vrapče, Zagreb, Croatia

2 Psychiatric Hospital Sveti Ivan, Zagreb, Croatia

3 Psychiatric Hospital Ugljan, Croatia

${ }^{4}$ Institute for Research and Development of the Sustainable Ecosystems (IRES), Zagreb,

5 University of Zagreb, School of Medicine

Differences in concentrations of bioelements may play a role in the pathogenesis of schizophrenia. In cross-sectional study which was conducted from May 2014 until May 2016 in University Hospital Vrapče and Psychiatric Hospital Sveti Ivan, Zagreb, Croatia, we compared the concentration of $43 \mathrm{BE}$ simultaneously in each sample of hair using Inductively coupled plasma mass spectrometry (ICP-MS) among 40 people suffering from schizophrenia and the control group of 42 people without psychiatric diagnosis, matched with the experimental group by age, sex and place of residence.

In hair samples of people with schizophrenia, significantly higher concentrations of manganese $(\mathrm{Mn})$ and silver $(\mathrm{Ag})$ were observed, and statistically significantly lower concentrations of zirconium $(\mathrm{Zr})$ and bismuth $(\mathrm{Bi})$.

The concentration of manganese in persons with schizophrenia was $0.134 \mathrm{u} / \mu \mathrm{g}$ (ppm) compared to the control group $0.0496 \mu \mathrm{g}$ (ppm). Everyone filled out a nutrition questionnaire and they were all coffee consumers.

Manganese is an essential trace mineral indispensable for bone formation and bone health in general. Dietary deficiencies are relatively rare as manganese is widespread in variety of plant foods, particulary in tea and coffee. Coffee cup contains: $6 \%$ RDA () Pantothenic Acid (Vitamin B5), 11\% RDA Riboflavin (Vitamin B2), 2\% RDA niacin (B3) and thiamine (BI), 3\% RDA potassium and manganese. Daily intake should be $10-20 \mathrm{mg}$. 1989. The FDA restricted the intake of caffeine in prescription drugs to $200 \mathrm{mg} /$ dose.

Caffeine is the most commonly used psychoactive drug in the world and acts in the same way as other stimulant medications such as amphetamines.

The former researches suggest that around five coffees, or the equivalent of $200 \mathrm{mg}$ of caffeine, may be enough to tip people over the edge and cause psychotic-like symptoms.
Caffeine has an effect on the central nervous system and cardiovascular system with metabolic effects and all depend on the amount taken. The average dose of caffeine is from 85 to $250 \mathrm{mg}$, which is about 1-3 cups of coffee, and the effect is a sense of availability, a reduced sense of fatigue, and an easy flow of thought. At higher doses, 250 - 500mg results in a feeling of restlessness, nervousness, insomnia and shyness. In high doses it can cause the so-called hyperadrenergic syndrome with cerebral attacks and cardiovascular instability.

Caffeine is in the blood for 5 minutes after taking, the highest blood concentration is 30 to 60 minutes. It is metabolised by the liver and disappears from the body on average from half an hour to 5-8 hours in healthy non-smokers (in the case of smokers the secretion is accelerated).

Caffeine-induced psychiatric disorders should be included in the differential diagnoses for patients presenting with psychotic symptoms. Overuse of coffee or other caffeine-containing beverage generally involve the symptoms of organic psychosis such as hallucinations, delusions, paranoia, and thought disorder. Other symptoms may include mania, erratic behavior, and aggression.

The question we can not answer right now is: Was the coffee intake responsible for elevated levels of manganese and positive symptoms? Do people with schizophrenia have elevated values regardless of dietary habits? The answer could be obtained by further monitoring of the same patients, who were still younger patients (25-35 years) in time when this research was conducted, knowing its toxicity in high doses and the neurological illnesses it may cause. 